\title{
A Wide Field Auroral Imager (WFAI) for low Earth orbit missions
}

\author{
N. P. Bannister ${ }^{1}$, E. J. Bunce ${ }^{1}$, S. W. H. Cowley ${ }^{1}$, R. Fairbend ${ }^{2}$, G. W. Fraser ${ }^{1}$, F. J. Hamilton ${ }^{1}$, J. S. Lapington ${ }^{1}$, \\ J. E. Lees ${ }^{1}$, M. Lester ${ }^{1}$, S. E. Milan ${ }^{1}$, J. F. Pearson ${ }^{1}$, G. J. Price ${ }^{1}$, and R. Willingale ${ }^{1}$ \\ ${ }^{1}$ Department of Physics \& Astronomy, University of Leicester, University Road, Leicester, LE1 7RH, UK \\ ${ }^{2}$ Photonis SAS, Avenue Roger Roncier, Brive, 19410 Cedex, France
}

Received: 6 January 2006 - Revised: 12 December 2006 - Accepted: 22 January 2007 - Published: 8 March 2007

\begin{abstract}
A comprehensive understanding of the solar wind interaction with Earth's coupled magnetosphere-ionosphere system requires an ability to observe the charged particle environment and auroral activity from the same platform, generating particle and photon image data which are matched in time and location. While unambiguous identification of the particles giving rise to the aurora requires a Low Earth Orbit satellite, obtaining adequate spatial coverage of aurorae with the relatively limited field of view of current space bourne auroral imaging systems requires much higher orbits. A goal for future satellite missions, therefore, is the development of compact, wide field-of-view optics permitting high spatial and temporal resolution ultraviolet imaging of the aurora from small spacecraft in low polar orbit. Microchannel plate optics offer a method of achieving the required performance. We describe a new, compact instrument design which can observe a wide field-of-view with the required spatial resolution. We report the focusing of $121.6 \mathrm{~nm}$ radiation using a spherically-slumped, square-pore microchannel plate with a focal length of $32 \mathrm{~mm}$ and an F number of 0.7. Measurements are compared with detailed ray-trace simulations of imaging performance. The angular resolution is $2.7 \pm 0.2^{\circ}$ for the prototype, corresponding to a footprint $\sim 33 \mathrm{~km}$ in diameter for an aurora altitude of $110 \mathrm{~km}$ and a spacecraft altitude of $800 \mathrm{~km}$. In preliminary analysis, a more recent optic has demonstrated a full width at half maximum of 5.0 \pm 0.3 arcminutes, corresponding to a footprint of $\sim 1 \mathrm{~km}$ from the same spacecraft altitude. We further report the imaging properties of a convex microchannel plate detector with planar resistive anode readout; this detector, whose active surface has a radius of curvature of only $100 \mathrm{~mm}$, is shown to meet the spatial resolution and sensitivity requirements of the new wide field auroral imager (WFAI).
\end{abstract}

Keywords. Magnetospheric physics (Instruments and techniques) - Radio science (Remote sensing)

Correspondence to: N. P. Bannister

(npb@star.le.ac.uk)

\section{Introduction}

Observations of the aurora (representing magnetic fieldmapped images of magnetospheric plasma populations), in combination with ionospheric flow monitoring from the ground and space, provide unique information on magnetospheric dynamics and are thus a powerful diagnostic for the way in which the solar wind influences auroral activity (Doe et al., 1997). Beginning with the Dynamics Explorer 1 satellite (Frank et al., 1981), several missions have obtained ultraviolet images of the auroral oval (as opposed to non-imaging measurements made on earlier missions). These observations can be combined with measurements from particle and field detectors and with ground-based radar data to derive a comprehensive understanding of the solar wind interaction with Earth's coupled magnetosphere-ionosphere system (e.g. Milan et al., 2003).

Auroral imaging missions typically occupy highly elliptical, high-altitude near-polar orbits to ensure efficient coverage of high latitudes. For example, the IMAGE spacecraft had an initial apogee of $\sim 45000 \mathrm{~km}$ over the Northern Hemisphere (Mende et al., 2000), and a perigee of $1000 \mathrm{~km}$. However, in-situ monitoring of the charged particles responsible for the production of auroral displays requires instrumentation in low Earth orbit (LEO); for example, the Fast Auroral Snapshot Explorer (FAST) was launched into a $351 \times 4175 \mathrm{~km}$ polar orbit (Carlson et al., 1998), while satellites of the Defense Meteorological Satellite Program (DMSP) are in $830 \mathrm{~km}$ circular near-polar orbits (see for example Rich et al., 1985).

The different orbits of particle detector and auroral imager spacecraft preclude complete correlation of data. Furthermore, current auroral imagers are unable to provide a simultaneous match to the coverage and spatial resolution of modern ground-based radar systems such as the Super Dual Auroral Radar Network "SuperDARN" (Greenwald et al., 1995) from LEO. Wide-field coverage requires the use of high altitudes, while high spatial resolution can only be achieved

Published by Copernicus GmbH on behalf of the European Geosciences Union. 


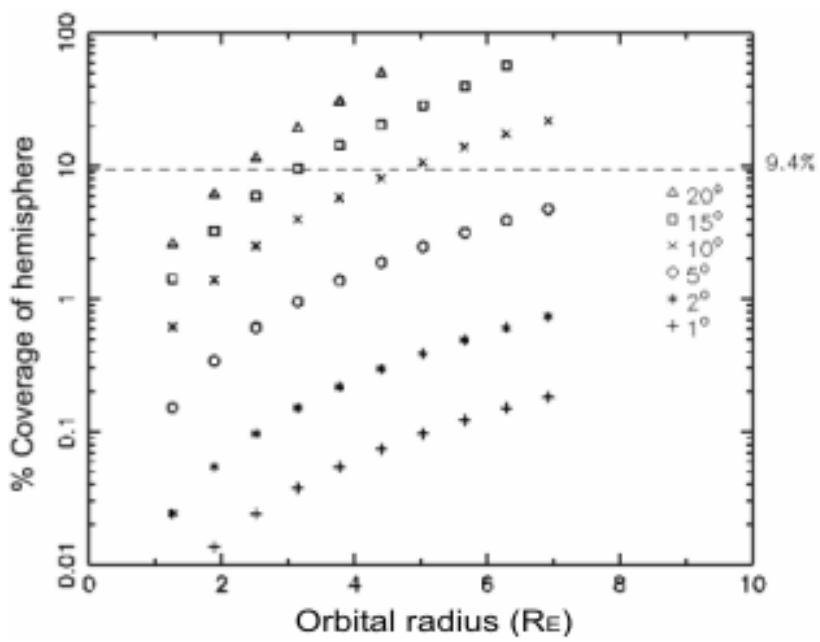

Fig. 1. Percentage coverage of a hemisphere of the Earth versus orbital radius for FOV values between $1^{\circ}$ and $20^{\circ}$. The dashed line indicates a coverage of $9.4 \%$, equivalent to the auroral oval (Ruohoniemi and Baker, 1998). Orbital radius is expressed in Earth radii, measured from the centre of the Earth.

using instruments at lower altitudes. Imaging the entire auroral oval requires a field of view (FOV) of $\sim 136^{\circ}$ at an altitude of $800 \mathrm{~km}$ above the pole (Ruohoniemi and Baker, 1998), and conventional optics fall significantly short of these requirements. For example, the Wideband Imaging Camera (WIC) onboard IMAGE has a $17^{\circ} \times 17^{\circ} \mathrm{FOV}$ and a spatial resolution of $52 \times 52 \mathrm{~km}$ per pixel at apogee, or $1.2 \times 1.2 \mathrm{~km}$ at perigee (Mende et al., 2000; Frey et al., 2001), while the ultraviolet imager on Freja (with a $600 \times 1700 \mathrm{~km}$ orbit) had a FOV of $22.4^{\circ} \times 30^{\circ}$ and a spatial resolution of $5 \mathrm{~km}$ at apogee (Murphree et al., 1994). Figure 1 shows the coverage of the accessible hemisphere of the Earth as a function of geocentric radial distance for FOV values between $1^{\circ}$ and $20^{\circ}$. It is clear that acceptable auroral coverage is only achievable, using conventional optics, from geocentric orbital radii greater than about $\sim 3.5 R_{E}$, at the expense of spatial resolution (and feasibility, given that the majority of mission opportunities are likely to be in LEO).

The goal of synoptic auroral imaging requires an innovative step in optical design. In this paper we describe a novel design for a wide FOV far ultraviolet (FUV) auroral imager based on microchannel plate (MCP) optics technology. The Wide Field Auroral Imager (WFAI), shown in Fig. 2, consists of several subsystems. Microchannel plate (MCP) optics focus a wide field, high resolution image of the aurora onto a detector consisting of a curved ("slumped") MCP electron multiplier and resistive anode readout, which records the position and arrival time of photons, allowing the scanning motion of the orbital platform to be removed from the image. Although the detector has negligible energy resolution, the use of appropriate filters allows quantitative observations in particular wavelength regions of interest. In the following

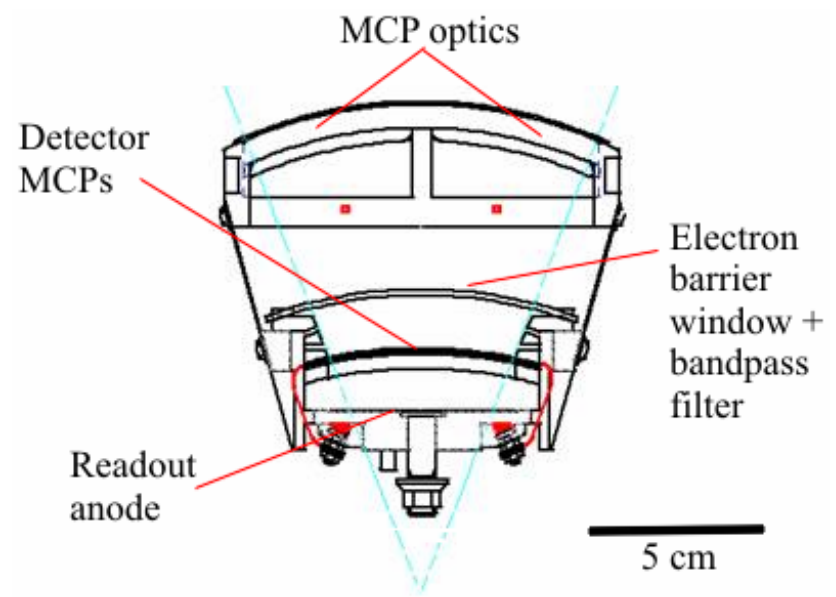

Fig. 2. WFAI module schematic. Note that planar windows and filters may be substituted for the curved elements shown here. Approximate scale indicated.

sections we discuss the function and principal design features of each subsystem. Section 2 describes the main requirements of an auroral imaging instrument, and in Sect. 3 we outline the performance characteristics of microchannel plate optics including an estimation of count rates expected for typical auroral observations. In Sect. 4 we describe the performance of a prototype MCP optic of $3 \mathrm{~cm}$ focal length, and in Sect. 5 we consider an MCP detector of pronounced convex curvature which is suitable for use in the instrument. Section 6 contains a brief consideration of module and instrument design, followed in Sect. 7 by a discussion of the results from the current programme and prospects for future instrument development. The next stages of our WFAI development programme are summarised in Sect. 8 .

\section{Instrument requirements}

\subsection{Science requirements}

The instrument design drivers are given in Table 1. Optical design and spacecraft orbital parameters should combine to make possible the instantaneous viewing of most of the auroral oval, while spatial resolution must be sufficient to resolve structure on scales better than $25 \mathrm{~km}$, providing adequate correlation with ionospheric flow measurements. Bright auroral features can exhibit horizontal velocities of up to several $\mathrm{km} \mathrm{s}^{-1}$; temporal resolution is therefore also an important factor. Conventional detectors, such as the intensified CCDs of the VIS instrument on Polar (Frank et al., 1995) are integrating devices, and thus provide no information about photon arrival time within a given frame time. In such a detector, the maximum integration time before blurring occurs is given by the ratio of instrument spatial resolution to spacecraft velocity, which, in polar LEO, is $\sim 7.5 \mathrm{~km} \mathrm{~s}^{-1}$. For a resolution 
Table 1. Performance requirements of a wide field FUV auroral imager in polar LEO.

\begin{tabular}{ll}
\hline Parameter & Requirement \\
\hline Spatial resolution & Less than $25 \mathrm{~km} \times 25 \mathrm{~km}$ footprint \\
Field of view for complete coverage & From $800 \mathrm{~km} \mathrm{LEO:} 130^{\circ} \times 130^{\circ}$ \\
& From $1 R_{E}$ altitude $(6378 \mathrm{~km}): 42^{\circ} \times 42^{\circ}$ \\
Temporal resolution & Better than $3 \mathrm{~s}$ \\
Bandpass & $140-180 \mathrm{~nm}$ band which contains the molecular N2 Lyman-Birge-Hopfield (LBH) \\
& emissions $(140-160 \mathrm{~nm}$ for the LBH short, and 160-180 nm for LBH long ranges) \\
Count rate & $<2.3 \mathrm{MHz}$ for $1 \mathrm{kR}$ feature (see Sect. 3.2) \\
\hline
\end{tabular}

of $25 \mathrm{~km}$, the maximum integration time is therefore $\sim 3 \mathrm{~s}$. In contrast, with the photon counting MCP detector described in Sect. 4 the arrival times of individual photons can be recorded with a precision which is better than $1 \mathrm{~ms}$ (e.g. Siegmund et al., 1997). Since the velocity vector of the spacecraft is well known, the instantaneous arrival position of the photon in the moving imager frame can be mapped back onto the Earth frame with an uncertainty given by the spacecraft velocity $V_{s c}$ multiplied by the instrument time resolution, $\tau$, which in this case is less than $7.5 \mathrm{~m}$. The effective limit on observation time for a stationary auroral feature is determined by how long that feature stays in the field of view of the imager.

The required angular resolution $(\Delta \theta)$ is determined from the minimum cell length $(L)$ on the Earth's surface for a given separation between spacecraft and object, $h$ :

$L=h \tan (\Delta \theta)$.

The distance along a line of sight to the aurora $(h)$ increases with the angle between the nadir and that line of sight. We can place limits on the angular resolution required by considering the extreme case of imaging the entire auroral oval from a position above the pole. This requires a FOV of $136^{\circ}$ and an angular resolution of $0.47^{\circ}$ for a cell of $25 \mathrm{~km}$ from an altitude of $800 \mathrm{~km}$, assuming the auroral emission occurs at $110 \mathrm{~km}$ altitude above the surface of the Earth. To image a $25 \mathrm{~km}$ auroral cell at nadir, the angular resolution required is $2.08^{\circ}$. As we discuss below, these performance specifications are achievable with MCP optics.

\subsection{Bandpass filters}

The primary goal of the WFAI is to provide high spatial and temporal resolution imaging in a global context (i.e. images of the entire auroral oval) at perigee in LEO. Although high spectral resolution is unnecessary to meet these goals, the addition of energy resolution increases the science which can be performed with the instrument. Since MCP detectors have negligible intrinsic energy resolution, adding this capability requires the introduction of filters to define specific bandpasses.

It has been shown with previous imagers that the ability to determine the ratio of one wavelength band to another (for instance between the "long" and "short" bands of the LymanBirge-Hopfield ( $\mathrm{LBH}$ ) $\mathrm{N}_{2}$ lines) can provide information on the depth of penetration of the particles giving rise to the aurora (e.g. Torr et al., 1995). This in turn provides an estimate of the energy spectrum of the particles, allowing the particle source region to be identified. To date, the most effective FUV filters for auroral imaging (in terms of their ability to provide narrow bandwidth and effective out-of-band blocking) are those produced for the FUV imager on board the Polar Mission. These sophisticated filters, described by $\mathrm{Zu}-$ kic et al. (1993), allow the isolation of specific wavebands of interest. Narrowband filters designed for observation of OI lines have a bandwidth of $<5 \mathrm{~nm}$, and a peak transmittance of $23.9 \%$ and $38.3 \%$ for filters with central wavelengths of 130.4 and $135.0 \mathrm{~nm}$, respectively, allowing quantitative measurements of the 130.4 and $135.6 \mathrm{~nm}$ lines of OI. Broadband filters for the FUV imager allow quantitative measurements of the LBH-s (140-160 nm) and LBH-l (160-180 nm) bands: one filter provides a bandpass centred at $150 \mathrm{~nm}$ with a bandwidth of $10 \mathrm{~nm}$, serving the LBH-s region; the other, with a central wavelength of $170 \mathrm{~nm}$ and a bandwidth of $11 \mathrm{~nm}$, is well matched to the LBH-l region. These highly selective filters allow the measurement of precipitating particle energies and interaction altitudes. A well-established method of determining these parameters involves the comparison of two emission lines, one of which is absorbed by the oxygen Schumann-Runge absorption continuum, as a function of altitude (e.g. Germany et al., 1998). For example, more energetic electrons penetrate deeper into the atmosphere before producing aurorae; photons emitted in the LBH-s are absorbed by $\mathrm{O}$ in the atmosphere, while photons in the $\mathrm{LBH}-$ 1 band are not. Hence the strength of the LBH-l emission can be used to indicate the power input to the system, while the ratio of the LBH-s to the LBH-l emission provides information on the altitude and energy of the auroral activity.

Although filters for the Polar-FUV imager are ideally matched to the diagnostic methods described above, they are reflection devices (multi-layer $\Pi$-stacks), used at a $45^{\circ}$ angle of incidence, in order to produce these highly specific wavebands. Reflection filters are incompatible with the very compact WFAI design, and are unsuitable for wide-field 
Table 2. Columns 1-3: transmission properties for filters appropriate to the WFAI instrument, based on those flown on the DE-1 mission. Columns are central wavelength, bandpass FWHM, and transmission at central wavelength. Columns $4 \& 5$ : estimated count rate for dayside auroral observations in each band, for a bare glass and CsI photocathode-equipped detector respectively, for a single WFAI module with $45.8^{\circ} \times 45.8^{\circ}$ field of view.

\begin{tabular}{lllll}
\hline$\lambda_{c}(\mathrm{~nm})$ & $\Delta \lambda(\mathrm{nm})$ & $T_{c}(\%)$ & $R_{\text {glass }}(\mathrm{cts} / \mathrm{s})$ & $R_{\mathrm{CsI}}(\mathrm{cts} / \mathrm{s})$ \\
\hline 156.0 & 34 & 18 & $1.62 \times 10^{6}$ & $3.99 \times 10^{7}$ \\
150.0 & 41 & 25 & $4.52 \times 10^{6}$ & $1.06 \times 10^{8}$ \\
154.0 & 43 & 17 & $2.67 \times 10^{6}$ & $6.32 \times 10^{7}$ \\
\hline
\end{tabular}

applications, where the broad range of input angles leads to a broadening of the filter bandpass. Filters for the WFAI must be used in transmission, and must be compatible with the wide field and large focal plane of the imaging device. $\mathrm{Zu}$ kic et al. (1993) review the development of filters for FUV applications, and find that previous designs used in transmission resulted in low throughput, and provided wavebands too large for the selectivity required to perform the diagnostics described above. In addition, some filters (e.g. those based on the Fabry-Pérot design) suffer from pass windows at longer wavelengths. This is particularly undesirable in the context of the WFAI, where global imaging of auroral emission requires observations in both the night-time and daylit hemispheres; in the latter case, long wavelength leakage will admit significant quantities of visible photons which will mask the FUV emission.

The lack of narrow bandwidth transmission filters with comprehensive out-of-band blocking characteristics precludes certain studies, such as measurement of precipitating particle energies and interaction altitudes described above. However, transmission devices are available which provide useful wavelength selectivity and which have a proven space heritage. Frank et al. (1981) describe filters used on the Dynamics Explorer Mission. These devices comprise both narrow-band interference filters and $\mathrm{BaF}_{2}, \mathrm{CaF}_{2}$ and $\mathrm{MgF}_{2}$ windows. Aluminium flashing is applied to the windows to suppress long wavelength transmission, allowing auroral observations in the sunlit ionosphere. Table 2 includes specifications for three DE-1 filters which are of particular interest in the context of the WFAI instrument, along with count rate estimates for each filter in the WFAI context (these estimates are discussed in Sect. 3.2). The design of these filters appears to be compatible with the requirements of the WFAI in that they are used in transmission, and can be produced in formats which are large enough to cover the WFAI focal plane.

The filters cover the LBH band, and are sufficiently opaque to Lyman $\alpha$ wavelengths so that, when combined with a window material such as $\mathrm{CaF}_{2}$, auroral emissions in the LBH band can be imaged in both the dark and sunlit iono- spheres. By restricting the WFAI to a single, broad bandpass covering the $\mathrm{LBH}$ emissions, the ability to measure the precipitating electron energies and distributions from direct measurements of the long- and short- LBH measurements is lost. However, quantitative measurements of electron impact excitation of $\mathrm{N}_{2}$ in dark and sunlit ionospheres becomes possible, and missions such as DE-1 and IMAGE have shown that such broadband measurements can produce valuable science (see e.g. Rairden et al., 1986; Frey et al., 2003).

\section{Microchannel plate optics}

\subsection{Overview}

To determine whether the proposed instrument can meet the scientific goals, we consider previous MCP optic results from Leicester and elsewhere, and the auroral imager performance which could be obtained with these MCPs. We describe the results of Monte Carlo ray trace simulations used to specify the MCP geometry (radius of curvature, channel width etc) for optimum FUV imaging in terms of effective area and angular resolution. We then estimate the sensitivity and maximum count rate expected from an MCP-based auroral imager in LEO.

An MCP optic consists of a spherically curved glass plate containing a very large number of channels of square cross section, mutually aligned with good regularity. The plate curvature is not introduced by grinding methods (as employed for conventional telescope mirrors and lenses) but is produced by bending or "slumping" the initially planar MCP onto a polished metal mandrel. This produces a different channel geometry to grinding: in the ground MCP, the pore axes are parallel, while in the slumped MCP the long axes of all the channels intersect at a distance $R_{\mathrm{MCP}}$ (the MCP radius of curvature) along the optical axis. A metallic layer (e.g. Ni) is deposited on the channel walls to enhance the reflectivity of the optic at X-ray energies. Recent studies have investigated alternative coating materials (e.g. Ir, Ru, Pt) (Nussey, 2005), and work on identifying the optimum coating for use in a wide field auroral imager (WFAI) such as that described here is ongoing.

Parallel beams of radiation are focused to a point by orthogonal reflections from the walls of the channels. The focal length of an MCP optic is $f=R_{\mathrm{MCP}} / 2$ (for a source at infinity) and the point spread function (PSF) is cruciform in nature (Fraser et al., 1993a), consisting of a central focus produced by odd numbers of reflections off orthogonal faces, accompanied by intersecting line foci which arise from single reflections. MCP optics have no "preferred axis", permitting much larger fields of view than those possible with conventional optical designs. MCP optics are the key technology for the Lobster imaging all-sky X-ray telescope (Fraser et al., 2002); here, they allow us to realise a compact, low mass instrument which meets the requirements of Table 1. In 
Table 3. MCP optic parameters considered in X-ray studies to date and in the present work.

\begin{tabular}{ll}
\hline Parameter & Demonstrated values \\
\hline Channel side length (D) & $8.5-200 \mu \mathrm{m}$ \\
Channel aspect ratio (L/D) & $50: 1-500: 1$ \\
Format & up to $54 \times 54 \mathrm{~mm}^{2}$ \\
Slump radius $\left(R_{\mathrm{MCP}}\right)$ & $0.07-1.0 \mathrm{~m}$ \\
Channel surface roughness & $1.1 \mathrm{~nm} \mathrm{rms}$ \\
Angular resolution & 7 arcminutes FHWM \\
Metallisation for enhanced reflectivity & $\mathrm{Ni}, \mathrm{Ir}$, or Ru \\
\hline
\end{tabular}

Table 3 we summarise the range of MCP parameters demonstrated in previous laboratory studies at the University of Leicester Space Research Centre (see, for example, Brunton et al., 1997, 1999; Fraser et al., 1994). The best angular resolution demonstrated in previous X-ray work ( $7^{\prime}$ full width at half maximum, or FWHM) is a factor of four better than the value required to provide a match to radar data, from LEO (see Sect. 2).

The bandpass over which a given MCP optic can focus is determined by its aspect ratio (channel length $L$ divided by channel width, $D$ ). Fraser et al. (1993a) describe the focusing of parallel beams of $0.1-2 \mathrm{keV} X$-rays, while results for hard X-ray focusing (50-65 keV) are presented by Price et al. (2002). The present paper is concerned with the performance of MCP optics in the FUV waveband defined by the bandpass filters discussed above. Since angular resolution $\Delta \theta$ is an important consideration, the MCP channel size $D$ must be chosen such that diffraction effects, which scale as $\lambda / D$, are negligible. For the $D=84 \mu \mathrm{m}$ channels of the prototype optic (Sect. 4), the diffraction contribution is only 4.9 arcsec FWHM at $121.6 \mathrm{~nm}$, which is insignificant compared to (a) the intrinsic geometrical resolution $D / f=5.8$ arcminutes, and (b) the target resolution of $2.08^{\circ}$ for $25 \mathrm{~km}$ cells at nadir from $800 \mathrm{~km}$ altitude.

\subsection{WFAI optic design and detector requirements}

A Monte-Carlo ray tracing model (Brunton et al., 1997) has been used to establish the optimal design parameters in terms of maximising optic effective area. In the model, a squarepore MCP optic with slump radius $R_{\mathrm{MCP}}=100 \mathrm{~mm}$ focuses light onto a spherically slumped MCP detector of the same radius. Although the ideal detector radius of curvature is half that of the optic, slumping a microchannel plate electron multiplier to only $50 \mathrm{~mm}$ radius is likely to present significant manufacturing difficulty. Adopting the larger radius of curvature will increase the FWHM of the point spread function by approximately $20 \%$ at the extreme edge of the field. The results of this modelling are presented in Table 4 . In this section we estimate the detector requirements of an instrument based on this ideal MCP optic, beginning with an estimation
Table 4. MCP optic properties adopted for the ray tracing study (the effective area is wavelength independent in the FUV band considered here).

\begin{tabular}{ll}
\hline Parameter & Dimension \\
\hline Channel width (D) & $100 \mu \mathrm{m}$ \\
Channel length (L) & $1000 \mu \mathrm{m}$ \\
Aspect ratio & $10: 1$ \\
Radius of curvature & $100 \mathrm{~mm}$ \\
Optic area & $40 \times 40 \mathrm{~mm}^{2}$ \\
Effective area & $1.64 \mathrm{~cm}^{2}$ \\
\hline
\end{tabular}

of the maximum count rate which can be sustained by the detector, followed by an estimation of the count rates expected for a typical auroral observation. The count rate generated in the instrument is dependent on the electron barrier, bandpass filter (these two roles being combined into a single element), and any QE-enhancing detector photocathode, as well as the optic itself. Filters and any photocathodes should be chosen such that the detected count rate does not exceed the count rate capacity of the detector when imaging a typical bright aurora.

The maximum achievable count rate in an MCP detector is limited by the charge replenishment time of the channels, which is related to the ratio of MCP output pulse current $I_{p}$ to strip current $I_{s}$ (i.e. the MCP conductance current flowing in the high resistivity surface conducting glass of the MCP structure). These quantities can be expressed as

$I_{p}=\bar{G} N$,

and

$I_{s}=\frac{V_{0}}{R_{c h}}$,

where $\bar{G}$ is the average charge gain of the detector, $N$ is the count rate per channel, $V_{0}$ is the bias voltage of the MCP and $R_{c h}$ is the effective resistance of a single microchannel (Fraser et al., 1991). Fraser et al. (1993b) model the operation of MCP detectors at high count rates, and provide data for the variation in limiting pulse current to strip current ratio (Fig. 1 in that work), as a function of the number of channels illuminated, for MCP detectors with channel sizes similar to those proposed for the WFAI. In the case of the MCP detector discussed here, the $12.5 \mu \mathrm{m}$ diameter channels are arranged in a hexagonal close pack structure with a pitch of $15 \mu \mathrm{m}$, and thus the channel number density is approximately $5.1 \times 10^{9} \mathrm{~m}^{-2}$. The illuminated area of the detector has a diameter of $40 \mathrm{~mm}$, so that there are approximately $6.4 \times 10^{6}$ illuminated channels. From Fig. 1 of Fraser et al. (1993b), the limiting ratio $I_{p} / I_{s}$ in this case is 


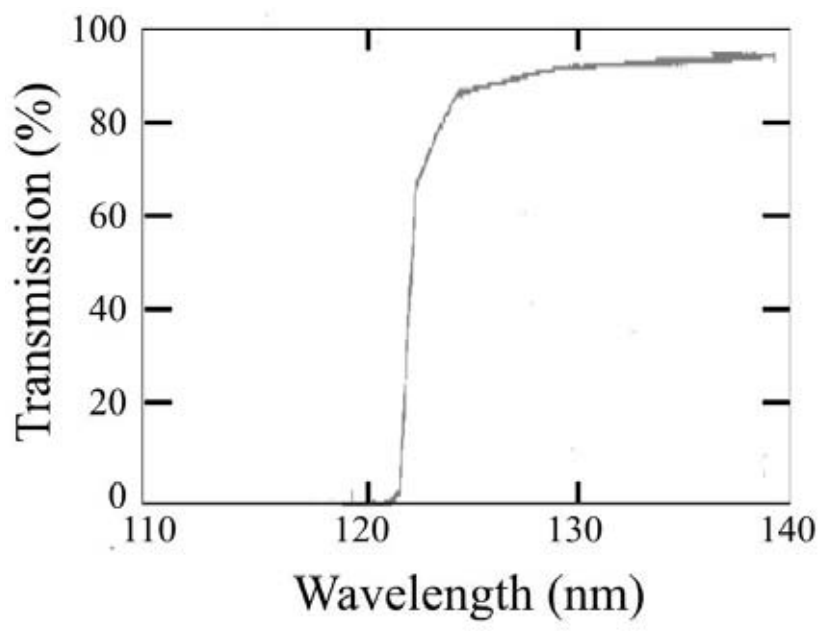

Fig. 3. Transmission curve for $0.5 \mathrm{~mm}$ thick $\mathrm{CaF}_{2}$ filter. Data from Laufer et al. (1965).

approximately 0.1. From Eqs. (2) and (3), the ratio $I_{p} / I_{s}$ can be expressed as

$$
\frac{I_{p}}{I_{s}}=\frac{\bar{G} N R_{c h}}{V_{0}} \approx 0.1
$$

We now make some conservative assumptions for the performance of the MCP to be used in the WFAI detector, adopting $V_{0}=1500 \mathrm{~V}, \bar{G}=1 \mathrm{pC}$ and $R_{\mathrm{mcp}}=40 \mathrm{M} \Omega$. Treating the MCP as $6.4 \times 10^{6}$ resistors in parallel, the resistance of an individual channel is $R_{c h}=2.6 \times 10^{14} \Omega$. Then from Eq. (4), the limiting count rate per channel is $N=0.6 \mathrm{cts} \mathrm{s}^{-1}$. Hence, for full field illumination we find a maximum count rate of approximately $4 \times 10^{6}$ counts $\mathrm{s}^{-1}$ can be sustained by the multiplier.

Auroral intensity (expressed in Rayleighs, where 1 Rayleigh corresponds to $10^{6}$ photons $\mathrm{s}^{-1} \mathrm{~cm}^{-2}$, emitting into $4 \pi \mathrm{sr}$ ), is conventionally quoted as the intensity of the strongest emission line within the spectrum (the $130.5 \mathrm{~nm}$ OI line). However, in the case of the WFAI, it is the integrated intensity over the range of wavelengths defined by the bandpass filter, which is of interest. Torr et al. (1995) provide data on the spectrum of the aurora (Fig. 2.4.1b and Table 2.6.1 in that work), and find that in the $140-170 \mathrm{~nm}$ band, the integrated intensity of the aurora is typically between 600-1200 Rayleighs, with the dayglow emission being of a similar intensity; in addition, there are strong lines of OI at 130.4 and $135.6 \mathrm{~nm}$ which fall within the bandpass of the filters considered here. We have estimated typical count rates which would be observed by an MCP optic-based auroral imager, assuming a total emission of 2400 Rayleighs in the observed waveband (dayglow and aurora), plus contributions from the OI lines at the maximum level quoted by Torr et al. (1995), i.e. 20000 and 2000 Rayleighs at $130.4 \mathrm{~nm}$ and $135.6 \mathrm{~nm}$, respectively. The count rate $R$ in a detector with quantum ef- ficiency (QE) $Q$ at the focus of an MCP optic with effective area $A$ in $\mathrm{cm}^{2}$ is (based on Eq. (3), Torr et al., 1995),

$R=\frac{10^{6}}{4 \pi} A \Omega t_{w} t_{f} Q I$,

where $\Omega$ is the solid angle subtended by the instrument field of view, $t_{w}$, is the transmission of the window (used to seal the detector against electrons - see Sect. 6.2), $t_{f}$, the transmission of the bandpass filter, and the brightness of the emission integrated over the wavelength range of interest is $I$ Rayleighs.

To estimate $R$ we adopt the wavelength-independent optic effective area of $1.64 \mathrm{~cm}^{2}$ determined from the Monte Carlo studies of a mechanically perfect optic with $\mathrm{L}: \mathrm{D}=10: 1$ (see Table 4). We consider the response of the instrument for the three filters listed in Table 2, and in each case adopt the wavelength-dependent $\mathrm{CaF}_{2}$ window transmission shown in Fig. 3. We have considered the response of the instrument based on a detector with (a) bare glass MCPs, and (b) a configuration in which the input face of the front MCP has a CsI photocathode $(\mathrm{KBr}$ photocathodes suffer from a minimum in the secondary electron yield at $170 \mathrm{~nm}$ and are therefore not considered). Simons et al. (1987) provide information on the QE of bare glass and CsI photocathode-equipped MCPs as a function of wavelength in the FUV region, and we assume these data here (efficiencies at these wavelengths are low e.g. at $160 \mathrm{~nm}, Q_{\text {bare }}=0.05 \%$ and $Q_{\mathrm{CsI}}=4 \%$ ). We find that if the source fills the entire field of view of a single module of the design described in Sect. 6 (i.e. a module consisting of 4 MCPs to generate a $45.8 \times 45.8^{\circ}$ (0.622 steradian) field of view satisfying the requirements outlined in Table 1), the resulting count rates range from $R_{\text {glass }}=1.62 \times 10^{6}$ counts $\mathrm{s}^{-1}$ for the first filter listed in Table 2 , to a maximum of $R_{\mathrm{CsI}}=1.06 \times 10^{8}$ counts $\mathrm{s}^{-1}$ in the case of the second filter. These rates are upper limits since the auroral intensity will not be uniformly bright in the field of view. Count rates are approximately halved for observations of the night-side ionosphere. The results are summarised in columns 4 and 5 of Table 2.

Detecting changes in the size of the "dim" region inside the auroral oval can give quantitative measurements of the magnetic reconnection rates at the dayside and in the magnetotail. This diagnostic is discussed by Milan et al. (2003). From Fig. 2 in that work we assume a factor of 40 difference between the brightest and faintest auroral emission to be imaged and hence the count rate for the dim region on the night-side, assuming a bare glass MCP and the first filter in Table 2 , is $R_{\text {glass }}=2 \times 10^{4}$ counts s ${ }^{-1}$. This compares to an intrinsic noise level of 0.1 counts $\mathrm{cm}^{-2} \mathrm{~s}^{-1}$ for a typical MCP detector, i.e. $<2$ counts $\mathrm{s}^{-1}$ for the sensitive area considered here.

While the processing of such large signals is not considered in this paper, it is apparent that bare glass MCP electron multipliers are capable of operation at the count rates 


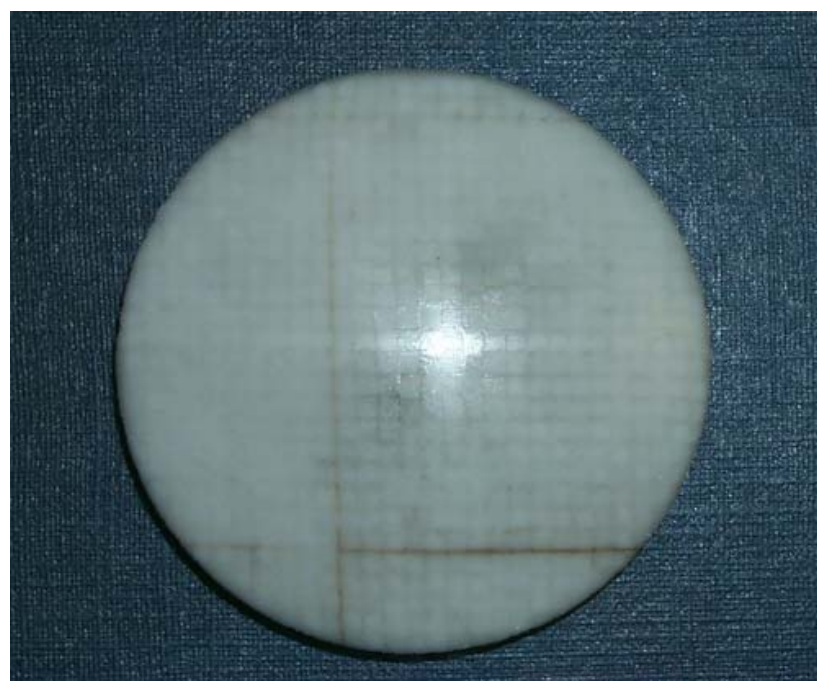

Fig. 4. The $46 \mathrm{~mm}$ diameter test optic. The squares seen on the surface are multifibre boundaries. The areas with more distinct discolouration contain more significant multifibre errors. The cruciform feature visible slightly right of centre is produced by light reflected from the channel walls.

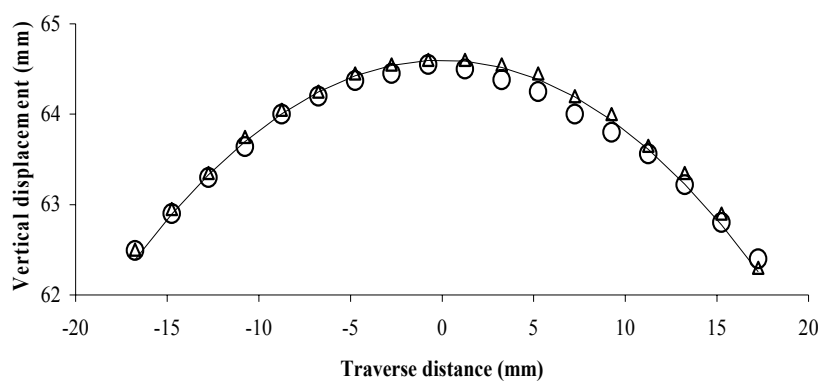

Fig. 5. Profile of test optic as determined by Talysurf profilometer. Open circles and triangles show measurements of the profile in orthogonal directions. Also shown (solid line) is the best fit circle to the profiles (radius $64.6 \mathrm{~mm}$ ). The maximum deviation of the optic profile from the best fit is of order $190 \mu \mathrm{m}$.

expected, and in addition have the benefit of simplifying detector manufacture and handling procedures.

\section{Performance of prototype MCP optics}

We have evaluated the performance of a prototype optic, manufactured by Photonis SAS. The MCP (shown in Fig. 4) was produced for a study into slumping procedures, and is not optimised for the current work. In particular, the $L / D$ value of 49:1 results in a lower effective area compared to the ideal $L / D$ discussed in Sect. 3. The MCP prototype is specified in Table 5.

The geometrical figure was characterised by two orthogonal scans of a Talysurf profilometer, and is shown in Fig. 5.
Table 5. Prototype MCP parameters.

\begin{tabular}{ll}
\hline Parameter & Value \\
\hline Outer diameter & $46.0 \pm 0.5 \mathrm{~mm}$ \\
Channel size $D$ & $84 \pm 2 \mu \mathrm{m}$ \\
Channel length $L$ & $4.2 \pm 0.1 \mathrm{~mm}$ \\
Channel pitch $P$ & $103 \pm 2 \mu \mathrm{m}$ \\
Radius of curvature $R_{\mathrm{MCP}}$ & $64.6 \pm 0.2 \mathrm{~mm}$ \\
Saggital depth & $4.5 \pm 0.2 \mathrm{~mm}$ \\
Theoretical open area fraction & $0.66 \pm 0.04$ \\
Coating & None \\
\hline
\end{tabular}

The MCP diameter was $46 \mathrm{~mm}$, and each traverse was limited by the deflection of the profilometer stylus, beginning and ending within $6 \mathrm{~mm}$ of the plate edge and passing within $\pm 0.5 \mathrm{~mm}$ of the centre. The best fit to the measured profile was a sphere with a $64.6 \pm 0.2 \mathrm{~mm}$ radius of curvature. Optical microscopy revealed significant distortion in the channel cross section and packing structures over approximately $5 \%$ of the MCP area. Figure 6 shows one such area (the black dots in the image are produced by particulate material in the microscope optics).

The optic was tested using the intense Lyman alpha UV emission at $121.6 \mathrm{~nm}$. Imaging tests were performed in the $27 \mathrm{~m}$ long optic test facility at Leicester (Price et al., 2002). UV light was generated using a deuterium lamp with a $\mathrm{MgF}_{2}$ window, producing an output continuum covering the range $112-400 \mathrm{~nm}$. Selection of the Ly- $\alpha$ line was achieved using a concave grating monochromator. The optic was mounted on a two-axis carriage located $20 \mathrm{~m}$ from the source, with its centre on the optical axis. The imaging detector (a $93 \times 93 \mathrm{~mm}^{2}$ active area, two-stage MCP detector with planar resistive anode readout) was initially placed $32.3 \mathrm{~mm}$ behind the optic, predicted to be the position of the optimum focus as determined by the MCP lens equation (Brunton et al., 1995),

$\frac{1}{l_{s}}-\frac{1}{l_{i}}=\frac{1}{f}$,

where $l_{s}$ is the distance from the source to the centre of the optic, and $l_{i}$ is the distance from the optic centre to the image (note that for an object at infinity, $f=R_{\mathrm{MCP}} / 2$ ). Due to the construction of the MCP support fixture, only the central $40 \mathrm{~mm}$ diameter of the optic was illuminated, with the outer rim being masked by the clamping assembly. Non-linearities in the resistive anode images were corrected in software.

Images generated with the Monte Carlo model were used to simulate the experimental results. Distortions in the channel wall and packing structure were incorporated into the model by multiplying a perfect optic structure with a matrix containing a Gaussian distribution of deviations in channel alignment of $1.47^{\circ}$ and $1.70^{\circ}$ about the nominal channel bias in $\mathrm{x}$ - and $\mathrm{y}$-axes, respectively. A comparison between the 


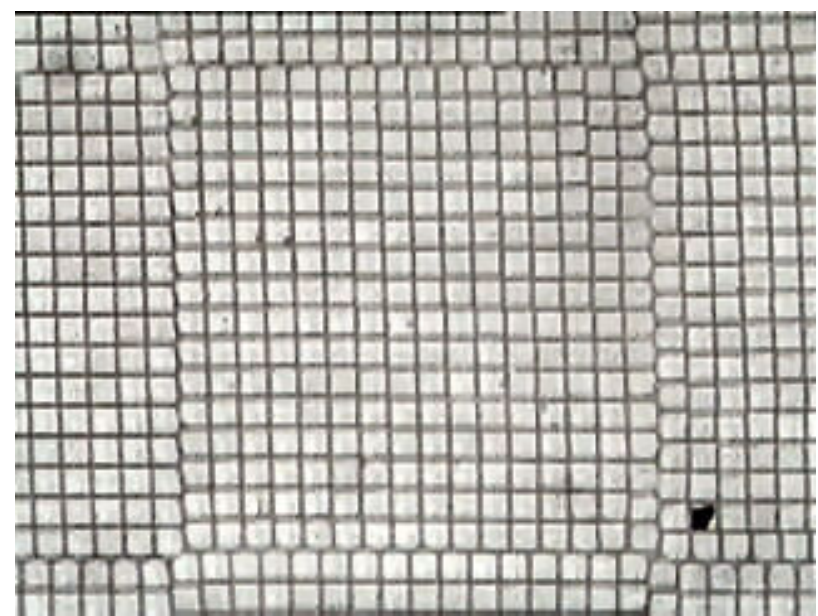

Fig. 6. Optical microscope image of the prototype MCP optic showing the channel structure. The channel side length is $84 \pm 2 \mu \mathrm{m}$.

calculated and observed performance of the optic is shown in Fig. 7. Truncation of the "west" and "south" cross-arms in the laboratory image is caused by deformation within the optic, specifically a twisting of the channels which is particularly evident in one quadrant of the MCP. The channel orientation in this section appears to be subject to random deviations from nominal alignment, resulting in the deformed cross-arms.

Despite differences between the quality of focus in the simulated and experimental images, good agreement is found between the predicted and measured focal lengths. The optimum focal distance (defined by a minimum in the FWHM of the central point spread function) occurs at an opticdetector separation of $38 \pm 1.5 \mathrm{~mm}$, compared to the theoretical FWHM minimum position of $32.3 \mathrm{~mm}$. The angular FWHM at best focus was measured to be $2.8^{\circ} \pm 0.1^{\circ}$, where the error is due to uncertainty in the detector-to-optic distance.

With the optic-detector separation set to $37 \pm 1 \mathrm{~mm}$ and the optic tilted about a vertical axis, images were taken at off-axis angles $\alpha$ covering the range $\pm 11.16^{\circ}$ in $2.23^{\circ}$ steps (Fig. 8). Since the focused light was known to pass through an angle of $2 \alpha$, the shift in position of the focal spot could be calculated. Over the total change in angle of $22.3^{\circ}$, the focus moved a distance of $32.3 \pm 1.4 \mathrm{~mm}$ (compared with a predicted distance of $28.9 \pm 1.7 \mathrm{~mm}$ ). These images confirm that the MCP optic focuses over a very wide FOV.

\section{Performance of a prototype imaging detector}

\subsection{Detector design}

The prototype detector consists of two $36 \mathrm{~mm}$ diameter MCPs with $12.5 \mu \mathrm{m}$ diameter pores, and a thickness of
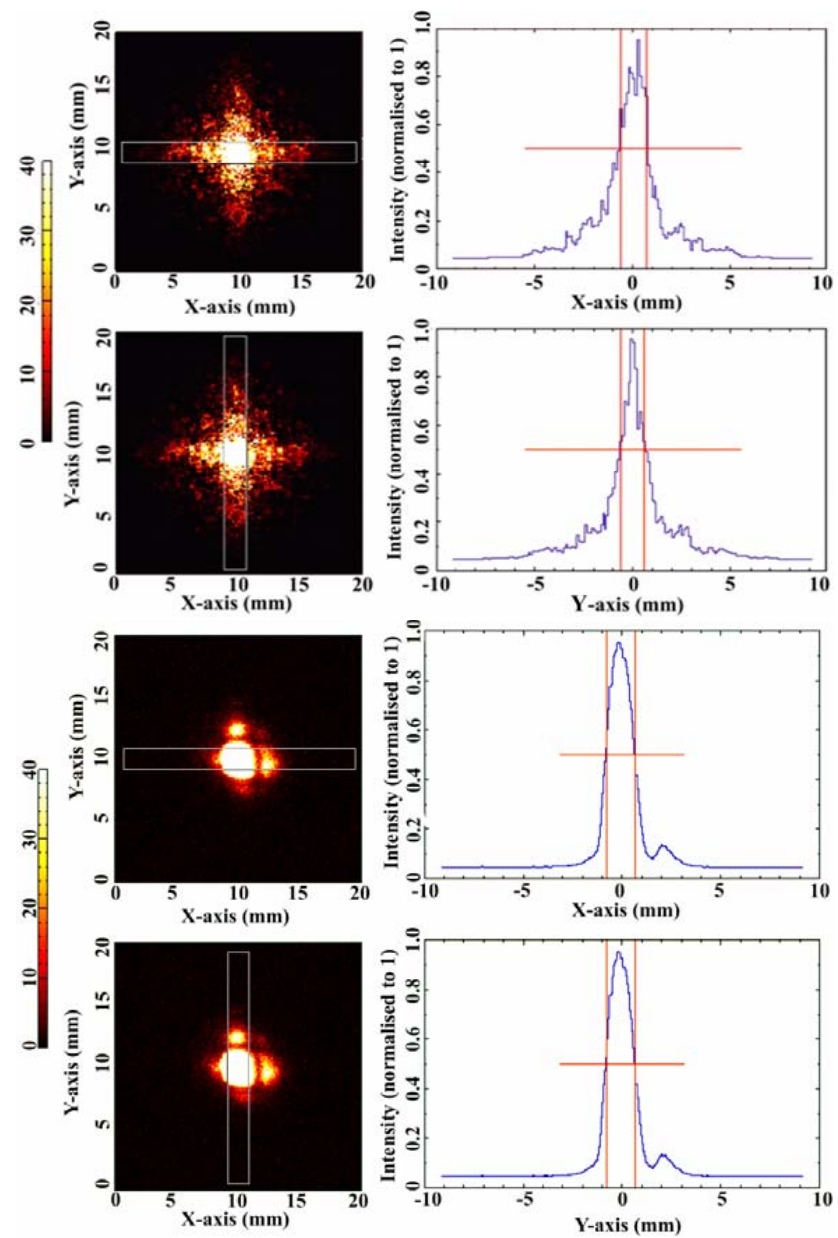

Fig. 7. Upper panel: Simulated optic performance at a wavelength of $121.6 \mathrm{~nm}$. Channel misalignments have been modelled by introducing an additional tilt to each channel. The size and direction of each tilt is taken from a Gaussian distribution with a FWHM of $1.47^{\circ}$ in the $\mathrm{x}$-axis and $1.70^{\circ}$ in the $\mathrm{y}$-axis. The resulting focal spot has a FWHM of $2.45^{\circ} \pm 0.2^{\circ}$ (horizontal) and $2.64^{\circ} \pm 0.2^{\circ}$ (vertical). Lower panel: laboratory measurements. Cross-arm structures characteristic of a square-pore MCP optic are clearly visible. The FWHM of the central focus is $2.38^{\circ}$ (horizontal) $\times 2.70^{\circ}$ (vertical) $\pm 0.22^{\circ}$.

$1.5 \mathrm{~mm}$. The MCPs are mounted one above the other in a "stack" such that the output charge pulse from the front MCP acts as the input pulse for the rear plate. To approximate the curved focal plane of an MCP optic, the plates in the detector are spherically slumped by the manufacturer (Photonis, Brive, France) to a radius of curvature of $100 \mathrm{~mm}$. Curved MCP detectors have a considerable space heritage. For example, a detector with $70 \mathrm{~mm}$ convex radius of curvature was used in the XUV telescopes for the ALEXIS satellite (Siegmund et al., 1990), and $165 \mathrm{~mm}$ concave MCPs were used for the ROSAT Wide Field Camera (Barstow and Sansom, 1990). However, the curved figures of these channel 
plates were produced by grinding the surface figure prior to channel etching. All the microchannels, therefore, were parallel in the ALEXIS and ROSAT missions. The slumping method adopted here is novel for such a small radius of curvature, having been used previously only for devices with much larger $R_{\mathrm{MCP}}$ values $-826 \mathrm{~mm}$ in the case of the Cosmic Origins Spectrograph for the Hubble Space Telescope (McPhate et al., 2000; Vallerga et al., 2002), and $875 \mathrm{~mm}$ for the Far Ultraviolet Spectrographic Explorer (FUSE) detectors (Siegmund et al., 1997).

In the present detector, the charge output from the rear MCP is imaged by a planar $52 \mathrm{~mm} \times 52 \mathrm{~mm}$ uniform resistive anode. The centre of curvature of the MCP lies behind the readout plane; the MCP-anode spacing is $5.9 \mathrm{~mm}$ and $3.6 \mathrm{~mm}$ at the MCP centre and edge respectively. The channels in one of the MCPs have axes which are perpendicular to the MCP surface - referred to as a " $0^{\circ}$ bias angle" plate. The other MCP contains channels which are inclined at $13^{\circ}$ with respect to the surface normal.

Although the MCP-anode distance varies because of the use of a slumped MCP, the readout system records the position of the centroid of each charge cloud, and in this respect the varying anode-MCP distance does not affect the "focus" of the detector. The variation in MCP-anode spacing affects the electric field at the rear of the detector, introducing nonlinearities in the resulting image. However, these distortions can be corrected by first quantifying nonlinearities in the image using a "pinhole mask" - a grid of small, regularly spaced holes in a sheet of material placed over the front MCP. The difference between measured and known pinhole location in the unprocessed image can then be used to correct for nonlinearities in the science frames, using processing in software. As well as performing this linearisation, variations in detector gain, pulse height distribution and quantum efficiency as a function of position were measured to identify the influence of the pore bias geometry.

The detector used in this work has been used to verify that the required detection efficiency and spatial resolution can be achieved. However, it should be noted that more sophisticated anode technology will be required for the WFAI instrument to accommodate the high count rates expected, while minimising the power requirements. The science requirements of a WFAI translate to a detector performance of $512 \times 512$ pixels at a global count rate of order $\sim 10^{6}$ count $/ \mathrm{s}$. The high count rate places several constraints on readout operation. Firstly, it is desirable to lower the gain of the MCP to reduce the "pore paralysis" time, which is a significant count rate limiting factor caused by the finite time taken to replenish electrons ejected into the channel during the avalanche process. The signal to noise ratio is reduced not only by the lower MCP gain, but by the shorter signal deadtime and faster shaping time required at these higher count rates. Secondly, the larger signal currents due to higher count rates can cause increased image instability in some traditional readouts such as the wedge and strip anode, an effect caused by

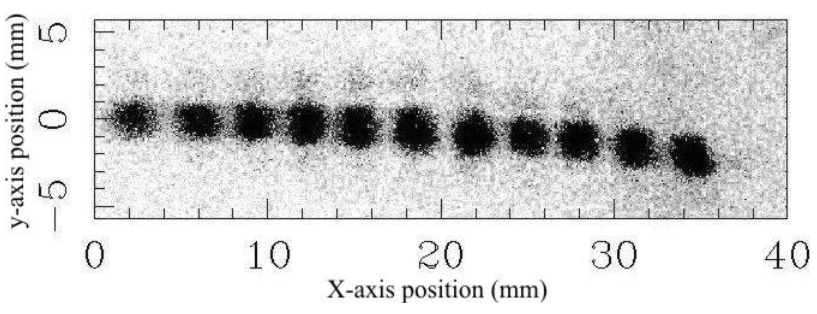

Fig. 8. Image sequence showing the shift in focus position across the imaging plane produced as the optic is tilted through an angle of $22.3^{\circ}$ in $2.23^{\circ}$ steps. The distance between adjacent spots is $2.89 \pm 0.08 \mathrm{~mm}$. The curved path followed by the spots may be due to local deviations from the spherical figure, and hence correct slump radius, of the MCP (see Fig. 5).

secondary electron redistribution between readout electrodes (Lapington, 1997) and mediated by DC offset voltages.

The latest developments in conductive image readout technologies (as described in Lapington et al., 2005) are required to accommodate the projected detector count rate. These devices utilize orthogonal strip electrodes with centroiding to achieve high spatial resolution, and high speed ASIC-based multichannel electronics to provide the improved signal to noise ratio necessary for operation at lower MCP gain. They are also designed to operate in the Image Charge mode, a technique in which signals are capacitively coupled to the electrodes, resulting in zero net DC signal current, eliminating DC offset voltages and providing excellent image stability.

\subsection{Results}

The detector was tested in two orientations, with the $13^{\circ}$ bias $\mathrm{MCP}$ at the input, $0^{\circ}$ bias MCP at the output in both instances to eliminate the effects of any intrinsic performance variation between the two MCPs. In the first instance the detector was tilted at $13^{\circ}$ with respect to the source direction, followed by an identical test at $0^{\circ}$ tilt. Figure 9 shows the uniformity of gain (the amount of charge produced at the readout by an incident photon) in both orientations. In the first case ( $13^{\circ}$ tilt) the pore axes at the centre of the MCP were parallel to the source direction, thus approximating the geometry of a $0^{\circ}$ bias front MCP. Figure 9 (left) shows that the gain at the centre of the image was lower than in the surrounding area, since photons arriving close to the centre of the detector interact at greater depth in the pores due to their small grazing angles. The mean MCP gain over the whole image is $4.06 \pm 0.4$ picoCoulombs (pC) in this configuration. Figure 9 (right) shows the same detector at $0^{\circ}$ tilt, such that the channel axes made an angle of $13^{\circ}$ with respect to the source direction. In this case, the mean gain is at $4.20 \pm 0.2 \mathrm{pC}$, and the uniformity of gain over the active area is improved. These results show that a detector for the WFAI should be fitted with a non-zero bias 


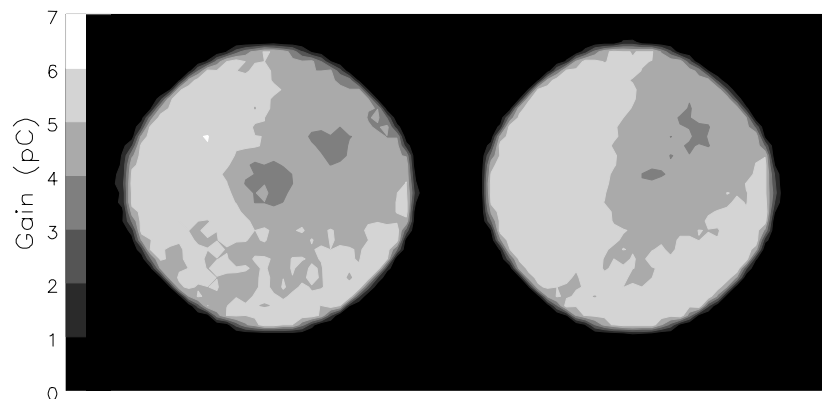

Fig. 9. Contour plots of the mean gain over the detector active area. Left: image acquired with the detector tilted by $13^{\circ}$ so that the effective pore bias angle at the centre of the image is $0^{\circ}$. Right: image acquired with the detector at normal incidence (i.e. MCP pores angled at $13^{\circ}$ to the source direction), showing improved gain in the centre of the active area.

angle MCP at the input to ensure minimal variation of gain over the imaging area.

Image resolution and linearity were measured by using a flat pinhole mask with $50 \mu \mathrm{m}$ diameter pinholes in a square array with a pitch of $0.5 \mathrm{~mm}$, placed in close proximity $(\sim 50 \mu \mathrm{m})$ to the input MCP, and illuminating the detector with $\mathrm{Cu}-\mathrm{L}(0.93 \mathrm{keV}) \mathrm{X}$-rays. Analysis of this data indicates a resolution of $\sim 31 \mu \mathrm{m}$ after deconvolution of the pinhole size and linearization of image distortions. When coupled to the optics specified in Table 4, this figure would correspond to a resolution of better than $0.5 \mathrm{~km}$ for nadir-directed observations of the aurora from $800 \mathrm{~km}$ LEO. It is therefore evident that the detector design described here is well matched to the optical design in terms of dynamic range, absolute sensitivity and image resolution, although as noted above, an alternative MCP geometry and readout technology would be chosen for a flight instrument to accommodate the high count rate requirements of the mission.

\section{Module design}

\subsection{Optical arrangement}

A single MCP optic of the type specified in Table 4 for the WFAI has a core FOV of $22.9^{\circ}$. Increasing the size of individual MCPs is not a feasible method of increasing the FOV, since a single large MCP optic cannot be slumped without introducing significant channel distortions. However, a wider FOV can be built up by tiling four MCPs in a $2 \times 2$ arrangement to approximate a spherically figured surface of the required size.

In the WFAI design proposed here, each module consists of a spherically figured surface made up of four smaller MCP optics, a bandpass filter, an electron barrier window, and a single slumped MCP detector. This arrangement, shown in Fig. 2, increases the FOV to $45.8^{\circ}$, and it is this configura-
Table 6. Maximum imaging time per feature as a result for three modular configurations. $\mathrm{R}_{\mathrm{MCP}}=100 \mathrm{~mm}$ for all optical elements, assuming a circular orbit at an altitude of $800 \mathrm{~km}$, and an auroral altitude of $110 \mathrm{~km}$.

\begin{tabular}{llll}
\hline Array size & Maximum FOV & $\begin{array}{l}\text { Time imaging the } \\
\text { aurora per orbit }\end{array}$ & $\begin{array}{l}\text { Imaging time } \\
\text { per feature }\end{array}$ \\
\hline 1 module & $45.8^{\circ} \times 45.8^{\circ}$ & $1183 \mathrm{~s}$ & $100 \mathrm{~s}$ \\
$2 \times 2$ modules & $91.6^{\circ} \times 91.6^{\circ}$ & $1354 \mathrm{~s}$ & $272 \mathrm{~s}$ \\
$3 \times 3$ modules & $137.4^{\circ} \times 137.4^{\circ}$ & $1996 \mathrm{~s}$ & $914 \mathrm{~s}$ \\
\hline
\end{tabular}

tion for which we provide count rate estimates in Sect. 3 . To achieve wider fields of view, an array of such modules is required, synthesizing FOV widths which are multiples of $45.8^{\circ}$ (Table 6). Note that each module in such arrangements will carry an MCP detector, so that the expected count rates from each detector will remain within the limits discussed in Sect. 3. Although "square" arrangements (e.g. $2 \times 2$ or $3 \times 3$ modules) provide a larger instantaneous field of view for three-axis stabilised platforms, a $1 \times 3$ module instrument could be used to reduce mass, cost and complexity (Fig. 10) for operation on a scanning platform. The two alternative pointing schemes for this WFAI spacecraft are illustrated in Fig. 11.

Table 6 also summarises the length of time that various WFAI configurations view a point in the auroral zone, assuming that the spacecraft vector is directed through the centre of a $5374 \mathrm{~km}$ diameter auroral oval, with the instrument fixed pointing to nadir such that it scans across the oval (the vertical distance from the aurora to the spacecraft was assumed to be $690 \mathrm{~km}$ ). While a single module with a $45.8^{\circ}$ FOV can observe a particular position for $100 \mathrm{~s}$ per orbit, significantly longer observations are possible by adding further modules to synthesise a wider field of view. With observation periods of several hundred seconds per orbit possible at time resolutions ranging from $3 \mathrm{~s}$ to tenths of seconds or better, the WFAI design will permit detailed studies of the dynamics of the aurora at very small spatial scales.

\subsection{Electron shielding}

A nadir-pointing auroral imager has to contend with a large upward-moving electron flux which must be effectively suppressed to minimise imager background. There are three inbuilt electron attenuation mechanisms in the WFAI design: (a) the low transmission of the MCP optics to a particle flux; (b) the conventional operation of the MCP detector with a high negative input potential $(\sim-4 \mathrm{kV})$ which renders the detector insensitive to electrons with energies below $4 \mathrm{keV}$, and (c) the stopping power of the $\mathrm{CaF}_{2}$ filter substrate which acts as a barrier to electrons and the intense Lyman- $\alpha(121.6 \mathrm{~nm})$ emission. Rees (1989) provides data for upward-moving electron intensities as a function of altitude (Fig. 3.2.4 in that 


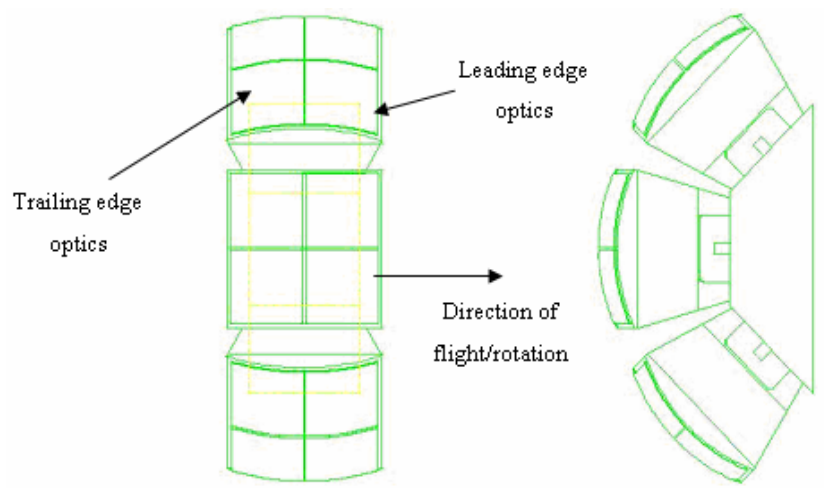

Fig. 10. Imager configuration for scanning the auroral oval, consisting of a $3 \times 1$ array of modules, each made up of $4 \mathrm{MCP}$ optic tiles in a $2 \times 2$ arrangement (as shown in Fig. 2).

work), based on data from the Dynamics Explorer-2 mission. The data show that electrons with energies in the range 4$20 \mathrm{keV}$ are of most concern for the current application. The extrapolated range $R\left(\mathrm{mg} \mathrm{cm}^{-2}\right)$ of an electron of energy $E$ $(\mathrm{MeV})$ in a solid is independent of atomic number and given by

$R=412 E^{1.265-0.0954 \ln E}(0.01<E<3)$,

(Zombeck, 1989). The density of $\mathrm{CaF}_{2}$ is $3.19 \mathrm{~g} \mathrm{~cm}^{-3}$. We obtain a range of only $2.13 \mu \mathrm{m}$ for $20 \mathrm{keV}$ electrons, and $42 \mu \mathrm{m}$ for $100 \mathrm{keV}$ electrons. These ranges should be compared with a likely $\mathrm{CaF}_{2}$ filter substrate thickness of at least $0.5 \mathrm{~mm}$ (as assumed in the sensitivity calculations above). Thus, total attenuation of the upward moving electron flux is achieved by the $\mathrm{CaF}_{2}$ alone. There is no requirement for either a magnetic or electrostatic particle diverter. Careful design of the detector housing will be required to ensure that there is no leak path for electrons around the filter, and to prevent the admission of photons generated by electron impact along the instrument optical path. In the latter case, the design of the optics (with channels $1 \mathrm{~mm}$ in length) minimises the surface area over which electrons can interact, and thus minimises the electron-generated photon flux.

\subsection{Detector resource requirements}

The power requirements of lower specification MCP detectors based on existing charge division readout technologies with flight qualified components, are typically $6.5 \mathrm{~W}$ (Lapington et al., 2003), representing the total power consumption of the detector system including the MCP high voltage supply, signal preamplifiers, shaping amplifiers, ADCs, FPGA-based digital processing and digital interfacing to the spacecraft bus. Power requirements for the generic technology discussed in Sect. 5.1, with its miniaturized, low power ASIC front-end electronics and integrated digital processing on FPGA, is expected to be similar. The estimated mass of
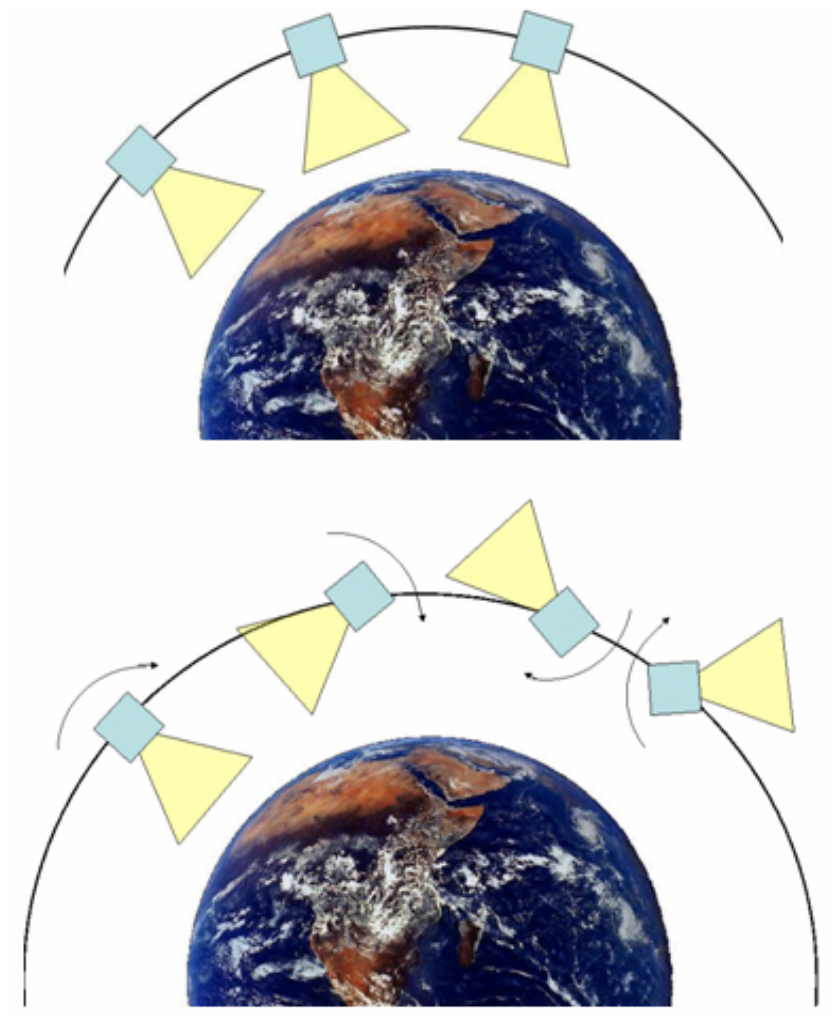

Fig. 11. Top: Imager mounted on a three-axis stabilised (despun) platform, with FOV directed toward nadir. Bottom: Spin stabilised spacecraft, imager FOV is scanned over Earth.

a single module is less than $1 \mathrm{~kg}$ (including optics, electronics and a $20 \%$ contingency). As a consequence of its compact low mass design, the WFAI is expected to be compatible with a wide range of future mission opportunities.

Due to the high intrinsic brightness of the auroral emission and the high resolution of the image, the data rate is likely to be high (approximately $80 \mathrm{Mbit} \mathrm{s}^{-1}$, including 10 bit digitisation each for $\mathrm{X}$ and $\mathrm{Y}$ positions, 6 bits for charge, and 8 bit for time encoding). On-board data compression schemes will therefore be an important element of the instrument design, and will be the subject of a future study.

\section{Discussion}

Despite obvious imperfections in the prototype which lead to distortions in the cross arms and a broadening of the focal spot, the FWHM of the true focus is $2.8^{\circ} \pm 0.1^{\circ}$, corresponding to a $39 \times 39 \mathrm{~km}( \pm 6.4 \mathrm{~km})$ footprint from an $800 \mathrm{~km}$ orbit. This figure places a lower limit on the performance possible from an MCP optic based imager, since the prototype optic was manufactured purely to illustrate the feasibility of slumping MCP optics to radii of only a few $\mathrm{cm}$. Extensive development work in optic production has been undertaken by Photonis SAS with funding from the European 


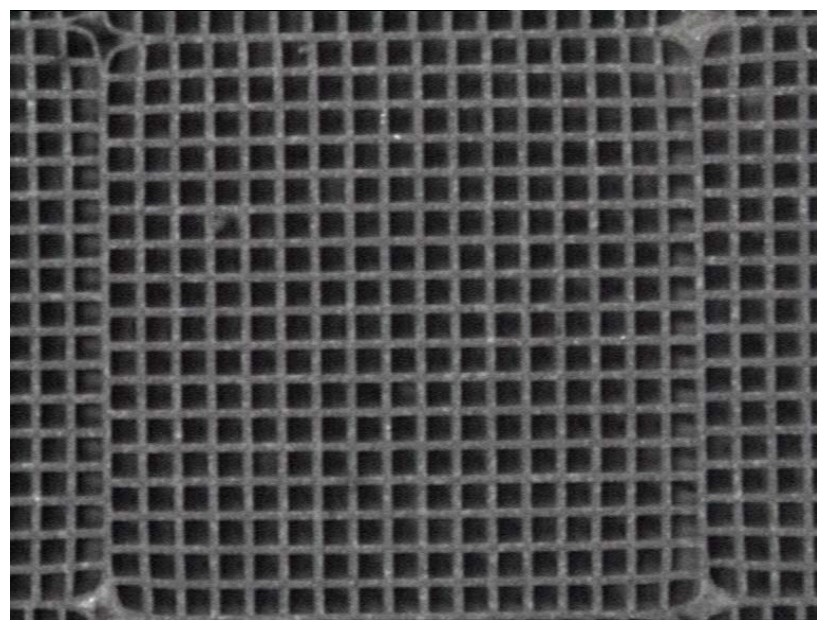

Fig. 12. Optical microscope image showing a single multifibre from a recently produced MCP optic. The individual channels have a width of $100 \mu \mathrm{m}$ and length of $1 \mathrm{~mm}$. The channel pitch (spacing) is $133 \mu \mathrm{m}$. Distortions are evident at the corner of the multifibre due to compression when the individual multifibres are fused together during manufacture. However, they occupy less than $1 \%$ of the total surface area and do not significantly affect the quality of focus.

Space Agency (see, for example, work reported by Bavdaz et al., 2004). We have recently received a batch of MCP optics produced for the WFAI development programme using the upgraded manufacturing facilities at Photonis. Early results suggest significant improvements in performance. Optical microscopy shows that the channel regularity in these MCPs is much improved compared to the prototype (see Fig. 12). $\mathrm{X}$-ray point-to-point focusing with a flat MCP from the new batch indicates that the line foci have a FWHM of 5.0 \pm 0.3 arcminutes, corresponding to a resolution of $1 \mathrm{~km}$ for auroral emission imaged at nadir from an altitude of $800 \mathrm{~km}$. Although errors in the figure introduced during the slumping process can be expected to degrade the FWHM value, the goal of $25 \times 25 \mathrm{~km}$ spatial resolution from $800 \mathrm{~km}$ is clearly achievable. We have also shown that slumped MCP, planar readout detector technology is capable of satisfying the sensitivity, dynamic range, focal plane curvature and spatial resolution requirements of the WFAI instrument.

This paper has been concerned with the performance of an auroral imager in polar LEO. Other mission concepts make use of highly elliptical orbits, with apogee in excess of $6 R_{E}$, from which the Earth's auroral oval subtends an angle of $3^{\circ}$. It is interesting to consider the potential of MCP optics at such altitudes, in the context of terrestrial missions and instruments deployed to Jupiter or Saturn, where high altitudes are required to image the extent of the auroral zones. The spatial resolution of our prototype optic corresponds to a cell size of $1670 \mathrm{~km}$ at $6 R_{E}$ - clearly insufficient to provide useful data for the studies outlined above. However, as we have noted, this prototype is not representative of the "state of the art" in MCP optic production. For the MCP defined in Table 4 , the resolution limit is $0.1^{\circ}$, corresponding to a cell size of $67 \mathrm{~km}$ at $6 R_{E}$, and the 5 arcminute resolution of the new flat MCP discussed briefly above corresponds to a cell size of approximately $56 \mathrm{~km}$ at the same distance. Further improvements are possible, since the theoretical limit to the spatial resolution of an MCP optic is determined by the angle subtended by a channel as viewed from the focal plane, or $D / f$, where $f$ is the focal length of the optic. By reducing the pore size to $35 \mu \mathrm{m}$, cell sizes of $27 \mathrm{~km}$ may be resolved from the same altitude. As noted in Table 3, MCP optics with channel diameters significantly smaller than this have been demonstrated in laboratory tests, so that high resolution imaging from considerable altitudes becomes possible with this technology. This optical performance, combined with the compact size and low mass of the instrument, provides, for the first time, the capability to perform high resolution wide field auroral imaging in both low and high altitude Earth orbits, and opens the way to practical, compact instruments which can be deployed on deep space missions to the outer planets.

\section{Future work}

In separate experiments, we have demonstrated the focusing of parallel beams of FUV radiation with a spatial resolution that satisfies the requirements of high resolution, wide field imaging in Low Earth Orbit (summarised in Table 1), and we have shown that an MCP detector can meet the goals of the WFAI concept in terms of dynamic range, absolute sensitivity and image resolution. We identify three key goals for the next phase of our development programme:

1. End-to-end testing of an MCP optic and WFAI-type detector. In the current work, data for FUV focusing were obtained using a standard laboratory detector. In the next phase of this work, an MCP optic will be coupled with the WFAI-type detector described in Sect. 5 to verify the results of our current work, showing that the endto-end performance of the instrument meets the goals of the WFAI concept.

2. Practical demonstration of MCP optic alignment. To generate the required field of view, a WFAI instrument will comprise four MCP optics tiled to form a single spherical surface as described in Sect. 6. These optical elements must be precisely aligned to ensure that the spatial resolution of the assembly preserves that of the individual elements (i.e. that the assembly performs as a larger, single MCP optic).

3. Demonstration of wide-band filter performance. The construction and performance (transmission, out-ofband blocking and response for wide angles of acceptance) of DE-1 type bandpass filters discussed in Sect. 2 
will be verified by introducing appropriate filter elements into the end-to-end demonstrator produced for task (1).

These tasks require the modification of our MCP detector and MCP optic laboratory facilities. This upgrading is currently in progress, and the results of the work packages summarised above will be presented in a future paper.

\section{Summary}

Microchannel plate optics offer a practical method for meeting the wide field of view and high spatial resolution requirements of future auroral imaging missions from LEO in a highly compact, low mass package. We have demonstrated the focussing of a parallel beam of Lyman-alpha UV light by a prototype $\mathrm{MCP}$ optic, and a preliminary design for a modular auroral imager based on this technology has been presented. The design of the instrument can be tailored to the nature of the spacecraft stabilisation method. In the case of a 3-axis stabilised platform, a number of modules are required to synthesise the entire field of view instantaneously. In the case of a spin-stabilised platform, a $1 \times 3$ module design can be used in combination with the scanning motion of the platform to achieve the required field.

An auroral imaging instrument based on this design is currently under consideration for the Chinese KuaFu-B spacecraft (Donovan et al., 2007' ; Tu et al., 2007).

Acknowledgements. This work was supported by PPARC grant PPA/G/0/2003/00013. We thank the Leicester Physics Department workshop for their efforts in upgrading the X-ray long beamline facility. F. J. Hamilton acknowledges the receipt of a Departmental studentship. S. W. H. Cowley was supported for part of this study by a Royal Society Leverhulme Trust Senior Research Fellowship. We thank the referees for useful comments provided during the preparation of this paper.

Topical Editor I. A. Daglis thanks two referees for their help in evaluating this paper.

\section{References}

Barstow, M. A. and Sansom, A. E.: The ROSAT WFC imaging detectors, Proc. SPIE, 1344, 244-254, 1990.

Bavdaz, M., Lumb, D., Peacock, A., Beijersbergen, M., and Kraft, S.: Status of x-ray optics development for the XEUS Mission, Proc. SPIE, 5488, 829-836, 2004.

Brittnacher, M., Spann, J., Parks, G., and Germany, G.: Auroral observations by the polar ultraviolet imager (UVI), Adv. Sp. Res., 20(4-5), 1037-1042, 1997.

\footnotetext{
${ }^{1}$ Donovan, E., Trondsen T., Spann, J., Liu, W., Spanswick, E., Lester, M., Tu, C.-Y., Ridley, A., Henderson, M., Immel, T., Syrjäsuo, M., Sofko, G., Cogger, L., Murphree, J., Rankin, R., Pulkkinen, T., Sigwarth, J., and Mende, S.: Global Auroral Imaging in the ILWS Era, Adv. Space Res., submitted, 2007.
}

Brunton, A. N., Fraser, G. W., Lees, J. E., Feller, W. B., and White, P. L.: X-ray focusing with $11 \mathrm{~mm}$ square pore microchannel plates, Proc. SPIE, 2159, 40-49, 1995.

Brunton, A. N., Fraser, G. W., Lees, J. E., and Turcu, I. C .E.: Metrology and modelling of microchannel plate X-ray optics, Appl. Opt., 36, 5461-5470, 1997.

Brunton, A. N., Martin, A. P., Fraser, G. W., and Feller, W. B.: A study of $8.5 \mu \mathrm{m}$ microchannel plate X-ray optics, Nucl. Instr. Meth., A431, 1, 356-365, 1999.

Carlson, C. W., Pfaff, R. F., and Watzin, J. G.: The Fast Auroral SnapshoT (FAST) mission, Geophys. Res. Lett., 25, 12, 2013 2016, 1998.

Doe, R. A., Kelly, J. D., Lummerzheim, D., Parks, G. K., Brittnacher, M. J., Germany, G. A., and Spann, J.: Initial comparison of POLAR UVI and Sondrestrom IS radar estimates for auroral electron energy flux, Geophys. Res. Lett., 24-8, 999-1002, 1997.

Frank, L. A., Craven, J. D., Ackerson, K. L., English, M. R., Eather, R. H., and Carovillano, R. L.: Global Auroral Imaging Instrumentation for the Dynamics Explorer Mission, Space Sci. Inst., 5, 369-393, 1981.

Frank, L. A., Sigwarth, J. B., Craven, J. D., Cravens, J. P., Dolan, J. S., Dvorsky, M. R., Hardebeck, P. K., Harvey, J. D., and Muller, D. W.: The visible imaging system (VIS) for the Polar spacecraft, Space Sci. Rev., 71, 297-328, 1995.

Fraser, G. W., Pain, M. T., Lees, J. E., and Pearson, J. F.: The operation of microchannel plates at high count rates, Nucl. Inst. Meth., A306, 247-260, 1991.

Fraser, G. W., Brunton, A. N., Lees, J. E., Pearson, J. F., and Feller, W. B.: X-ray focusing using square-pore microchannel plates First observation of cruxiform image structure, Nucl. Inst. Meth., A324, 404-407, 1993a.

Fraser, G. W., Pain, M. T., and Lees, J. E.: Microchannel plate operation at high count rates: further studies, Nucl. Inst. Meth., A327, 328-336, 1993b.

Fraser, G. W., Brunton, A. N., Lees, J. E., Pearson, J. F., Willingale, R., Emberson, D. L., Feller, W. B., Stedman, M., and Haycocks, J.: Development of microchannel plate (MCP) X-ray optics, Proc. SPIE, 2011, 215-226, 1994.

Fraser, G. W., Brunton, A. N., Bannister, N. P., Pearson, J. F., Ward, M., Stevenson, T. J., Watson, D. J., Warwick, R., Whitehead, S., O’Brian, P., White, N., Jahoda, K., Black, K., Hunter, S. D., Deines-Jones, P., Priedhorsky, W. C., Brumby, S. P., Borozdin, K. N., Vestrand, T., Fabian, A. C., Nugent, K. A., Peele, A. G., Irving, T. H., Price, S., Eckersley, S., Renouf, I., Smith, M., Parmar, A. N., McHardy, I. M., Uttley, P., and Lawrence, A.: LOBSTERISS: an imaging $\mathrm{X}$-ray all-sky monitor for the International Space Station, Proc. SPIE, 4497, 115-126, 2002.

Frey, H. U., Mende, S. B., Carlson, C. W., Gerard, J.-C., Hubert, B., Spann, J., Gladstone, R., and Immel, T. J.: The electron and proton aurora as seen by IMAGE-FUV and FAST, Geophys. Res. Lett., 28(6), 1135-1138, 2001.

Germany, G. A., Parks, G. K., Brittnacher, M. J., Spann, J. F., Cumnock, J., Lummerzheim, D., Rich, F., and Richards, P. G.: Energy Characterization of a Dynamic Auroral Event Using GGS UVI Images, in: Geospace Mass and Energy Flow: Results from the International Solar-Terrestrial Physics Program, edited by: Horwitz, J., Gallagher, D., and Peterson, W., AGU, 143-148, 1998.

Greenwald, R. A., Baker, K. B., Dudeney, J. R., Pinnock, M., Jones, T. B., Thomas, E. C., Villain, J.-P., Cerisier, J.-C., Senior, C., 
Hanuise, C., Hunsucker, R. D., Sofko, G., Koehler, J., Nielsen, E., Pellinen, R., Walker, A. D. M., Sato, N., and Yamagishi, H.: DARN/SuperDARN - a global view of the dynamics of highlatitude convection, Space Sci. Rev., 71, 761-796, 1995.

Lapington, J. S.: The effects of secondary electron emission on the operation of position sensitive anodes, Nucl. Instrum. Meth., A392, 336-340, 1997.

Lapington, J. S., Chakrabarti, S., Cook, T., Goeke, R. F., Gsell, J. C., and Gsell, V. T.: A Detector System for SPIDR - A Mission to perform Spectroscopy and Photometry of the IGM's Diffuse radiation, Proc. SPIE, 4854, 593-601, 2003.

Lapington, J. S.: Developments in high count rate microchannel plate detectors, Proc. SPIE, 5898, 85-94, 2005.

Laufer, A. H., Pirog, J. A., and McNesby, J. R.: Effect of Temperature on the Vacuum Ultraviolet Transmittance of Lithium Fluoride, Calcium Fluoride, Barium Fluoride, and Sapphire, J. Opt. Soc. Am., 55, 64-66, 1965.

McPhate, J. B., Siegmund, O. H. W., Gaines, G., Vallerga, J. V., and Hull, J.: The Cosmic Origins Spectrograph FUV Detector, Proc. SPIE, 4139, 25-33, 2000.

Mende, S. B., Heetderks, H., Frey, H. U., Lampton, M., Gellar, S. P., Abiad, R., Siegmund, O. H. W., Tremsin, A. S., Spann, J., Dougani, H., Fuselier, S. A., Magoncelli, A. L., Bumala, M. B., Murphree, S., and Trondsen, T.: Far Ultraviolet Imaging from the IMAGE Spacecraft: 2. Wideband FUV Imaging, Space Sci. Rev., 91(1-2), 271-285, 2000.

Milan, S. E., Lester, M., Cowley, S. W. H., Oksavik, K., Brittnacher, M., Greenwald, R. A., Sofko, G., and Villain, J.-P.: Variations in the polar cap area during two substorm cycles, Ann. Geophys., 21, 1121-1140, 2003, http://www.ann-geophys.net/21/1121/2003/.

Murphree, J. S., King, R. A., Payne, T., Smith, K., Reid, D., Adema, J., Gordon, B., and Wlochowicz, R.: The Freja ultraviolet imager, Space Sci. Rev., 70, 421-446, 1994.

Nussey, J.: Terrestrial and Space-based Applications of Microchannel Plate X-ray Optics, University of Leicester, Ph.D. thesis, 2005.

Price, G. J., Brunton, A. N., Fraser, G. W., Bavdaz, M., Beijersbergen, M. W., Boutot, J.-P., Fairbend, R., Flyckt, S.-O., Peacock, A., and Tomaselli, E.: Hard X-ray imaging with microchannel plate optics, Nucl. Inst. Meth., A490, 290-298, 2002.
Rees, M. H.: Physics and chemistry of the upper atmosphere, Cambridge University Press, 1989.

Rich, F. J., Hardy, D. A., and Gussenhoven, M. S.: Enhanced ionosphere-magnetosphere data from the DMSP satellites, EOS (ISSN 0096-3941), 66, 513-514, 1985.

Ruohoniemi, J. M. and Baker, K. B.: Large-scale imaging of highlatitude convection with Super Duel Auroral Radar Network HF radar observations, J. Geophys. Res., 103, 20 797-20 811, 1998.

Siegmund, O. H. W., Cully, S., Warren, J., Gaines, G. A., Priedhorsky, W., and Bloch, J.: Highly curved microchannel plates, Proc.SPIE, 1344, 346-354, 1990.

Siegmund, O. H. W., Gummin, M., Stock, J., Naletto, G., Gaines, G., Raffanti, R., Hull, J., Abiad, R., Rodriguez-Bell, T., Magoncelli, T., Jelinsky, P., Donakowski, W., and Kromer, K.: Performance of the double delay line microchannel plate detectors for the Far Ultraviolet Spectroscopic Explorer, Proc.SPIE, 3114, 283-294, 1997.

Simons, D. G., Fraser, G. W., de Korte, P. A. J., Pearson, J. F., and de Jong, L.: UV and XUV quantum detection efficiencies of CsIcoated microchannel plates, NIM A, 261, 579-586, 1987.

Torr, M. R., Torr, D. G., Zukic, M., Johnson, R. B., Ajello, J., Banks, P., Clark, K., Cole, K., Keffer, C., Parks, G., Tsurutani, B., and Spann, J.: A Far Ultraviolet Imager for the International Solar-Terrestrial Physics Mission, Space Sci. Rev., 71, 329-383, 1995.

Tremsin, A. S. and Siegmund, O. H. W.: UV Radiation Resistance and Solar Blindness of CsI and $\mathrm{KBr}$ Photocathodes, IEEE Trans. Nucl. Sci., 48(3), 421-425, 2001.

Tu, C.-Y., Schwenn, R., Donovan, E., Marsch, E., Wang, J.-S., Xia, L.-D., and Zhang, Y.-W.: Space Weather Explorer - The KuaFu Mission. Adv. Space Res., in press, 2007.

Vallerga, J. Zaninovich, J., Welsh, B., Siegmund, O., McPhate, J., Hull, J., Gaines, G., and Buzasi D.: The FUV detector for the cosmic origins spectrograph on the Hubble Space Telescope, Nucl. Inst. Meth., A477, 551-555, 2002.

Zombeck, M. V.: Handbook of Space Astronomy and Astrophysics, Cambridge University Press, 1989.

Zukic, M., Torr, D. G., Kim, J., Spann, J. F., and Torr, M. R.: Filters for the International Solar Terrestrial Physics Mission farultraviolet imager, Opt. Eng., 32, 3069-3074, 1993. 